\title{
Verzeichnis der Diagramme
}

Diagramm 1: Verhältnis "Geschäftsvolumen des Bankensektors zu Bruttosozialprodukt" 1960 - 1990 in \% ..........................................................5

Diagramm 2: Umweltbedingungen und Elemente der Bankpolitik ..................6

Diagramm 3: Rechtsangleichung im europäischen Binnenmarkt - Bankenrecht

Diagramm 4: Rechtsangleichung im europäischen Binnenmarkt - Börsenund Investmentrecht............................................................................43

Diagramm 5: Organe der Deutschen Bundesbank .............................................46

Diagramm 6: Segmente des Geldmarktes...........................................................64

Diagramm 7: Umlauf von Rentenwerten nach Emittentengruppen 1990.....68

Diagramm 8: Anlegerstruktur am Renten- und Aktienmarkt (1986) .............71

Diagramm 9: DTB-Teilnehmerstruktur ................................................................73

Diagramm 10: Eigenkapitalquote der deutschen Banken 1960 - 1990 (in \% des Geschäftsvolumens) ....................................................80

Diagramm 11: Anzahl der Mitarbeiter und Personalkosten im Büro...............84

Diagramm 12: Terminal-Installationen ................................................................106

Diagramm 13: Mitarbeiter der FIDUCIA ......................................................107

Diagramm 14: Maschinenkonfiguration der FIDUCIA in Karlsruhe.............108

Diagramm 15: Datentransport Bank - Rechenzentrum .....................................109

Diagramm 16: Genos-Transaktionen 1989 .......................................................110

Diagramm 17: Bankengruppen in Deutschland ..................................................119

Diagramm 18: Geschäftsstruktur ausgewählter Bankengruppen - Aktiva (Stand Ende 1990)

Diagramm 19: Geschäftsstruktur ausgewählter Bankengruppen - Passiva (Stand Ende 1990)

Diagramm 20: Marktanteile der einzelnen Bankengruppen am Geschäftsvolumen 1960-1990

Diagramm 21: Entwicklung der Marktanteile an den giralen Verfügungen auf Nichtbanken-Konten 
Diagramm 22: Entwicklung der Marktanteile am Zinsüberschuß $1970-1990$

Diagramm 23: Entwicklung der Marktanteile am Provisionsüberschuß 1970 1990

Diagramm 24: Entwicklung des Provisionsūberschusses im Verhältnis zum Zinsüberschuß

Diagramm 25: Entwicklung der Marktanteile am Betriebsergebnis $1970-1990$

Diagramm 26: Institutionen des paramonetären Bereichs

Diagramm 27: Entwicklung der Bilanzsumme der Bausparkassen und der Marktanteile 1970 - 1990

Diagramm 28: Entwicklung der eingezahlten Bausparbeträge und der Baudarlehen 1970 - 1990

Diagramm 29: Fondsvermögen der deutschen Wertpapierfonds 1970 - 1990147

Diagramm 30: Anteile der einzelnen Fondsarten am Mittelaufkommen 1970 1990

Diagramm 31: Fondsvermögen der deutschen Wertpapierfonds und der offenen Immobilienfonds (Ende 1990; in Mio. DM)

Diagramm 32: Beitragseinnahmen der Lebensversicherungsunternehmen in \% der Geldvermögensbildung der privaten Haushalte $1970-1990$

Diagramm 33: Entwicklung des Leasing-Anteiles an den volkswirtschaftlichen Investitionen 1975 - 1990 .154

Diagramm 34: Factoringumsätze in Deutschland 1980 -1990 .157

Diagramm 35: Kartentypen und Kartensysteme 160

Diagramm 36: Kundengruppenorientiertes Zielsystem einer Bank 182

Diagramm 37: Hierarchisch aufgebautes Zielsystem einer Bank. 183

Diagramm 38: Verhaltensgitter nach Blake und Mouton. . .184

Diagramm 39: 3-D Modell von Reddin. .185

Diagramm 40: Situative Führungstheorie nach Hersey und Blanchard. 186

Diagramm 41: Bedürfnispyramide . .193

Diagramm 42: Kontenrahmen für private Banken 197

Diagramm 43: Formblatt zum Jahresabschluß. . .199

Diagramm 44: Gewinn- und Verlustrechnung (Staffelform) 201 
Diagramm 45: Gewinn- und Verlustrechnung (Kontoform) .202

Diagramm 46: Schema eines Betriebsabrechnungsbogens (Kostenstellenrechnung)

Diagramm 47: Beispiel einer Stärken-Schwächen Analyse mit Konkurrenzvergleich

Diagramm 48: Komponenten der strategischen Situationsanalyse .220

Diagramm 49: Ableitung von Zukunftsszenarien .221

Diagramm 50: Positionierung Strategischer Geschäftsfelder in einer NeunFelder-Matrix. .223

Diagramm 51: Darstellung einer Cross-Impact Analyse .224

Diagramm 52: Darstellung der Planungslücken .226

Diagramm 53: Implementierung strategischer Planungssysteme bei Kreditinstituten im deutschsprachigen Raum 1970-1987. 237

Diagramm 54: Anknüpfungspunkte der operativen Ergebnisplanung .242

Diagramm 55: Informationssystem und Seja-Teilsysteme .254

Diagramm 56: Kostenrechnungs- und Kalkulationssystem................................255

Diagramm 57: Prognose und Finanzplanungssystem .........................................256

Diagramm 58: Vorgesehene Erweiterungen des Prognosesystems.................257

Diagramm 59: MIS-Report Nr. 4 ...........................................................................259

Diagramm 60: MIS-Report Nr. 1/2 .......................................................................260

Diagramm 61: Lageplan des Verwaltunggebäudes der Deutschen Bank......266

Diagramm 62: Nutzungsplan des Hauses und Aufzugskonzept (Taunusanlage 12) .267

Diagramm 63: Grundriß eines Normalgeschosses (Taunusanlage 12) ...........268

Diagramm 64: Geschäftsstelle Baden der Kreissparkasse Verden...................271

Diagramm 65: Kundenhalle der Volksbank Bassum eG.....................................272

Diagramm 66: Geschäftsstelle Merscheid der Stadt-Sparkasse Solingen......273

Diagramm 67: Aufbau eines EDV-Systems........................................................275

Diagramm 68: Entwicklung des SWIFT-Nachrichtenvolumens.........................281

Diagramm 69: Die SWIFT II - Systemarchitektur..............................................282

Diagramm 70: Bestimmungsgründe des zukünftigen Personalbedarfs (Gesamtbank) 
Diagramm 71: Tätigkeitenprofil im Bürobetrieb

Diagramm 72: Bestimmungsgründe des quantitativen Personalbedarfes für repititive Tätigkeiten

Diagramm 73: Verrichtungszeit pro Mitarbeiter .288

Diagramm 74: Maximales und minimales Anforderungsprofil eines Gruppenleiters der Kreditabteilung und Eignungsprofil eines Stellenbewerbers. .291

Diagramm 74: (Fortsetzung) .292

Diagramm 74: (Fortsetzung) .293

Diagramm 75: Verfahren der Arbeitsplatzbewertung .297

Diagramm 76: Strukturmerkmale des Marktbereiches von Banken. .304

Diagramm 77: Aufbauorganisation (Modell a) .306

Diagramm 78: Aufbauorganisation (Modell b) .307

Diagramm 79: Aufbauorganisation (Modell c) .308

Diagramm 80: Aufbauorganisation (Modell d - Zweigstelle) .309

Diagramm 81: Aufbauorganisation (Modell e) .310

Diagramm 82: Imageprofile des Schalterpersonals von drei Kreditinstituten322 Diagramm 83: Typologie nach Geldtypen und Kundensegmenten 326

Diagramm 84: Positionierung der Zielgruppen im 3D-Portfolio. .329

Diagramm 85: Einzugsbereich einer Bankstelle (Flächendiagramm) 332

Diagramm 86: Einzugsbereich einer Bankstelle (Ringzonen) .333

Diagramm 87: Marktkennziffern und Bankstellendichte alternativer Standorte (1-15 alternative Standorte für Zweigstellengründungen).....336

Diagramm 88: Entwicklung der Habenzinsen, des Diskontsatzes und des Preisindex 1980 - 1990. .348

Diagramm 89: Entwicklung der Habenzinsen und des Diskontsatzes 1989/90 (Monatswerte)

Diagramm 90: Entwicklung der Sollzinsen, des Diskontsatzes und der Umlaufsrendite 1980 -1990.

Diagramm 91: Entwicklung der Sollzinsen, des Diskontsatzes und der Umlaufsrendite 1989/90 (Monatswerte)

Diagramm 92: Imageprofile der Banken $X$ und $Y$. 